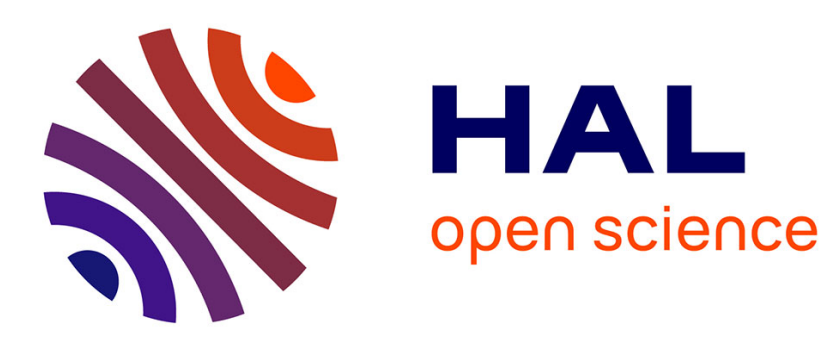

\title{
On the approximation of total absorption of the street open ceiling at low frequencies
}

\author{
Adrien Pelat, Bertrand Lihoreau
}

\section{To cite this version:}

Adrien Pelat, Bertrand Lihoreau. On the approximation of total absorption of the street open ceiling at low frequencies. Applied Acoustics, 2012, 73 (8), pp.734-740. 10.1016/j.apacoust.2012.02.006 . hal-02453261

\section{HAL Id: hal-02453261 \\ https://hal-univ-lemans.archives-ouvertes.fr/hal-02453261}

Submitted on 15 Dec 2020

HAL is a multi-disciplinary open access archive for the deposit and dissemination of scientific research documents, whether they are published or not. The documents may come from teaching and research institutions in France or abroad, or from public or private research centers.
L'archive ouverte pluridisciplinaire HAL, est destinée au dépôt et à la diffusion de documents scientifiques de niveau recherche, publiés ou non, émanant des établissements d'enseignement et de recherche français ou étrangers, des laboratoires publics ou privés. 


\title{
On the approximation of total absorption of the street open ceiling at low frequencies
}

\author{
Adrien Pelat, Bertrand Lihoreau \\ LAUM, CNRS, Université du Maine, avenue Olivier Messiaen, 72085 Le Mans, France.
}

\begin{abstract}
This work deals with numerical modeling of sound propagation in street canyons with flat building facades. The street is seen as an open waveguide and two 3D wave models are used : a parabolic equation model and a modal expansion model. The comparison between the models shows a very good agreement. Then, the study focuses on the radiation condition at the opening of the street. In usual energetic approaches as ray tracing, the opening is assumed to be perfectly absorbing. This assumption is realistic at high frequencies, however the reflection phenomenon caused by the geometric discontinuity at the opening is still an open question at low frequencies. This possible reflection is investigated through three parametric studies of the acoustic longitudinal energy flux decay along the street. The first study shows that the approximation of total absorption at the street open ceiling is no longer valid when the ratio $\eta$ between the street width and the wavelength is small. The second study shows that the effect of source height is weak except under restrictive conditions: when $\eta$ is small and when the source height is not small compared to the wavelength, the approximation of total absorption is acceptable for an elevated source. At least, the results of the last study show that the error made by assuming a perfectly absorbing ceiling is not negligible compared to the error made by considering perfectly reflecting walls.
\end{abstract}

Keywords: street canyon, numerical modeling, wave approaches, open ceiling reflection, guided waves.

Email addresses: adrien.pelat@univ-lemans.fr (Adrien Pelat), bertrand.lihoreau@univ-lemans.fr (Bertrand Lihoreau) 


\section{Introduction}

In modeling the sound propagation within an urban street canyon, a particular attention must be paid to description of the effects of the street open ceiling toward an infinite domain, the sky [1]. Basically, the opening leads to radiation losses: the waves, partially confined within the street while they propagate, are also radiated to the sky. As a consequence, the radiation losses contribute to the sound pressure level decrease with the distance to the source, in addition to the atmospheric losses and other dissipation phenomenon (absorption at walls for example). This work aims at investigating the attenuation caused by radiation losses at low frequencies. Even if the annoyance at low frequencies is caused by many types of sources, one of the main origin of its annoyance is the traffic flow. Indeed, Jonasson [2] and Can et al. (Fig. 6, 7, 8 and 9 of [3]) have shown that the noise caused by traffic flow is dominant in the $63 \mathrm{~Hz}$ octave band, particularly at low vehicle speeds. Can et al. also showed that the level at several locations within the urban environment are about 75-80 dB SPL. For such a sound level range, using A-weighting filters [4], that strongly attenuate the level in the $63 \mathrm{~Hz}$ bandwidth, is not adapted since it is only valid for weak levels ( $L \leq 40 \mathrm{~dB}$ $\mathrm{SPL}$ ). B-weighting or $\mathrm{C}$-weighting filters, that much less attenuates the low frequencies, are preferable. As a consequence, the sound levels at low frequencies and their perceived annoyance by people may be of great importance and predicting the low frequency propagation in the urban context is relevant.

To address the problem, the street is seen as an open waveguide. Indeed, the typical elongated shape promotes guided waves along the axis of the street. In contrast, the opening of this guide on an infinite domain - the sky - results in wave radiation. Several formulations have been proposed to model the sound propagation within 3D street canyons. The most used are so called energetic approaches as ray tracing [5], image-sources [6], radiosity based method [7] or sound particles [8], and are implemented in predictive software for acoustical engineering studies. In these approaches, the street open ceiling is modeled as a perfectly absorbing boundary. Hence, the geometry of the problem is equivalent to that of Fig. 1(a) where the height of the street above the ground is infinite. In the following, this geometrical configuration will be called "U-shaped" geometry. The assumption of total absorption at the street open ceiling seems to be well adapted in the high frequency domain. However, one may question the validity of this assump- 
tion in the low frequency domain. Notably, one can refer to the behavior of a duct in which the standing waves are due to the almost total reflection at the open extremity.

To investigate this question, we propose to compare the U-shaped geometry in Fig. 1(a) with the "T-shaped" geometry of Fig. 1(b) where no assumption is made on the behavior of the wave at the street open ceiling $(z=d)$. In this configuration, the street is seen as an uniform waveguide with a rectangular cross-section open on the infinite half space above, bounded by PML [9] to truncate the computational domain.

Regarding the frequency range of interest, wave-based methods are well adapted. Recently, several time-domain wave-based methods have been developed in the urban acoustic area $[10,11]$. As the phenomenon investigated here are time independent, methods in the frequency domain are preferable. In this article, we use two different wave approaches in the frequency domain. On the one hand, the parabolic equation method which has been successfully used to study outdoor, long range, propagation problems has recently been extended to model the propagation in a 3D open waveguide [12]. On the other hand, the modal expansion, used in the 70's [13] to describe the propagation within a street seen as a guiding structure between two infinite parallel planes has also recently been extended to the case of 3D open waveguides by using leaky modes [14]. This paper also aims at comparing both methods.

Section 2 recalls the theoretical background of both wave approaches. Section 3 presents the numerical results. First, the two methods are compared. Then, the assumption of total absorption is examined through three parametric studies of the leakage of the street. These studies respectively focus on the effects of the street width, the effects of the source height and the effects of absorbing walls and ground. Finally, conclusions and possible improvements to this work are drawn in Section 4. 


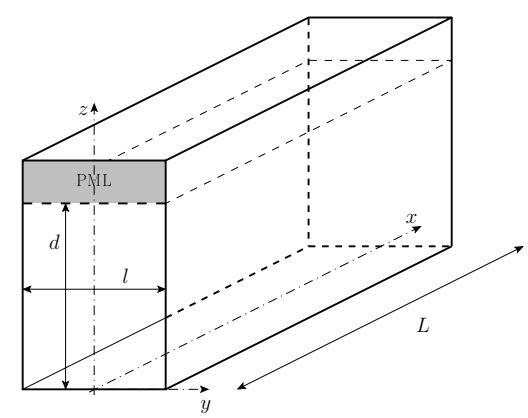

(a)

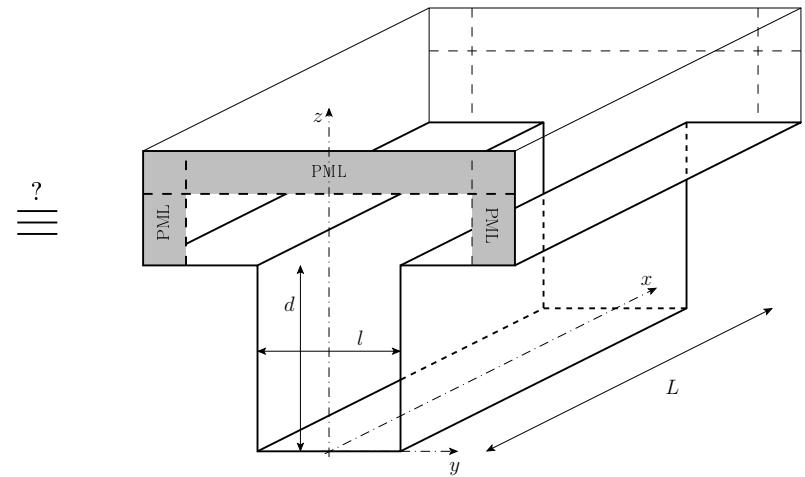

(b)

Figure 1: (a) The street is seen as a U-shaped uniform waveguide, of width $l$ and height $d$, where a perfectly absorbing ceiling, modeled by a PML, is considered $(z=d)$. (b) In the "T-shaped" geometry, the street is seen as an open uniform waveguide with a rectangular cross-section of same dimensions open on the infinite half space.

\section{Two wave approaches for the sound propagation modeling in a $3 \mathrm{D}$ uniform open canyon}

In both formulations, the equation to solve is the classic wave equation :

$$
\left[\frac{\partial^{2}}{\partial x^{2}}+\frac{\partial^{2}}{\partial y^{2}}+\frac{\partial^{2}}{\partial z^{2}}-\frac{1}{c^{2}} \frac{\partial^{2}}{\partial t^{2}}\right] p^{\prime}(x, y, z, t)=0,
$$

where $p^{\prime}$ is the acoustic pressure and $c$ the wave speed. In the frequency domain and taking into account the PML to bound the sky above the street, the wave equation to solve is

$$
\left[\frac{\partial^{2}}{\partial x^{2}}+\frac{1}{\tau} \frac{\partial}{\partial y}\left(\frac{1}{\tau} \frac{\partial}{\partial y}\right)+\frac{1}{\tau} \frac{\partial}{\partial z}\left(\frac{1}{\tau} \frac{\partial}{\partial z}\right)+k^{2}\right] p(x, y, z)=0,
$$

where $p$ is the complex acoustic pressure $\left(p^{\prime}=p e^{-i \omega t}\right), k$ is the wave number and $\tau$ is a complex scalar PML parameter fulfilling

$$
\begin{cases}\tau=A e^{\mathrm{j} \gamma} & \text { in the PML domain, } \\ \tau=1 & \text { in the physical domain }\end{cases}
$$

with $A>0$ and $\gamma \in] 0, \pi / 2[$. The boundary conditions are defined through 
an admittance parameter $\beta$

$$
\partial_{n} p+\mathrm{j} k \beta p=0
$$

with $\partial_{n}$ the normal derivative. The Neuman boundary conditions $\partial_{n} p=0$ are defined for the rigid outer borders of the PML.

\subsection{Parabolic equation}

In the PE models [15], Eq. (2) is splitted into two equations for the outgoing wave $(+)$ and the incoming wave $(-)$. The equation for the outgoing wave is:

$$
\left[\frac{\partial}{\partial x}-i k Q\right] p^{+}=0,
$$

where $Q$ is the operator defined by

$$
Q^{2}=1+\frac{1}{k^{2}}\left[\frac{1}{\tau} \frac{\partial}{\partial y}\left(\frac{1}{\tau} \frac{\partial}{\partial y}\right)+\frac{1}{\tau} \frac{\partial}{\partial z}\left(\frac{1}{\tau} \frac{\partial}{\partial z}\right)\right] .
$$

As the uniform canyon is assumed to be infinitely long, there is no coupling between outgoing and incoming waves and Eq. (5) represents an exact equation of the evolution of the acoustic pressure. The solution of Eq. (5) is :

$$
\phi(x+d x, z)=e^{i k(Q-1) d x} \phi(x, z),
$$

where $\phi=p^{+} e^{-i k x}$ is a scaled variable. The square root operator $Q$ is approximated using a Padé series expansion [16]. The previous initial- and boundary- value problem is numerically solved using an alternating direction method [17]. Eq. (7) is discretized with a standard finite difference method in height (z-axis) and width ( $y$-axis) and a Crank-Nicholson scheme is implemented as a marching algorithm ( $x$-axis).

\subsection{Coupled modal-finite element method}

In the coupled modal-finite element method, the modes of the canyon defined in the transverse domain $(y, z)$ are numerically found by using a standard FEM. Then, the acoustic field is computed by propagating these modes along the canyon:

$$
p(x, y, z)=\sum_{n \geq 1} P_{n} e^{\mathrm{j} k_{n} x} \phi_{n}(y, z)
$$


with $k_{n}=\sqrt{k^{2}-\alpha_{n}^{2}}, \alpha_{n}$ and $\phi_{n}(y, z)$ are respectively the eigenvalues and eigenfunctions of the numerical modes, only depending on the geometry of the street cross-section. $P_{n}$ are the modal amplitudes obtained by the development of the source on the modal basis. For details about the Modal-FE method see [18]. Three examples of such modes are represented in Fig. 2.

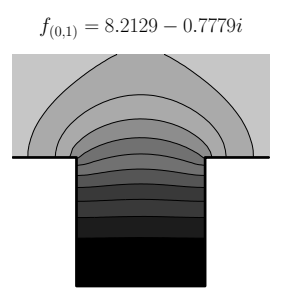

(a)

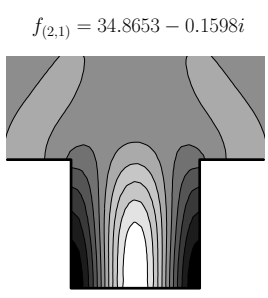

(b)

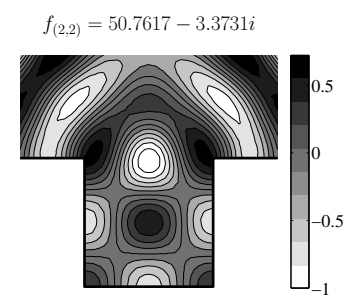

(c)

Figure 2: Three examples of leaky modes represented (real part) in the street cross-section. (a) and (b) modes highly confined within the street. (c) Mode with a more radiating pattern.

Owing to radiation losses above the street, the eigenvalues $\alpha_{n}$ of the leaky modes are complex. Then, whatever the source wave number $k$, the modal axial wave numbers $k_{n}$ are also complex. As a consequence, every mode is propagating and exponentially decreasing along the street (Eq. (8)).

Some modes are highly confined within the street cross-section (Fig. 2(a) and (b)) and are associated to $\alpha_{n}$ with a weak imaginary part. Others modes as the one shown in Fig. 2(c) display much more radiated patterns above the street and are associated to $\alpha_{n}$ with a larger imaginary part. Hence, at a given frequency, the mode of Fig. 2(c) will be much more leaky than modes of Fig. 2(a) and (b).

\section{Numerical Results}

\subsection{Parameters of the study}

In both methods, the acoustic field is computed downstream from a starting field $p(x=0, y, z)=p_{0}$, function of the transverse coordinates $(y, z)$, defined in the street input plane $(x=0)$. In the following results, the starter 
is chosen as an incident Gaussian beam given by:

$$
p_{0}=e^{-\frac{\left(y-y_{s}\right)^{2}+\left(z-z_{s}\right)^{2}}{2 \sigma^{2}}}
$$

where $y_{s}$ and $z_{s}$ are the position of the center of the Gaussian beam and $\sigma$ is a parameter controlling its width. This kind of starter can be seen as an approximation of the far field pattern generated by a point source. Nevertheless, the radiation pattern of a Gaussian beam is not omnidirectional, the directivity being driven by the divergence of the beam given by $\Theta \simeq 2 c / \pi f \sigma$ rad.

In the numerical results presented below, the fixed parameters are the height of the street $d=10 \mathrm{~m}$ and the center position of the Gaussian beam chosen as $\left(y_{s}, z_{s}\right)=(0,0.1) \mathrm{m}$ to approach typical source positions as vehicles (except for section 3.4 where the source height varies). The varying parameters are then the width of the street $l=5,10,20 \mathrm{~m}$ and the frequency of the Gaussian beam $f=62.5,125,250 \mathrm{~Hz}$.To compare the results with each other at different frequencies, we adjust $\sigma$ so that $\Theta$ remains constant $(\sigma=1.44$ for $f=125 \mathrm{~Hz}$ ). This choice for $\sigma$ is the result of a compromise which makes the source i) sufficiently spread out at $f=250 \mathrm{~Hz}(\sigma=0.72)$ in order to be well discretized, ii) sufficiently narrrow at $f=62.5 \mathrm{~Hz}(\sigma=2.88)$ to provide a non trivial source corresponding to an incident plane wave.

The main purpose of this work is to study the energy losses through the street open ceiling. These radiation losses can be evaluated by measuring the decrease of the longitudinal energy flux along the street. Hence, we define the integrated indicator $W(x)$, as:

$$
W(x)=\frac{1}{S} \int_{0}^{d} \int_{-l / 2}^{l / 2} \frac{1}{2} \mathbb{R}\left\{p v^{*}\right\} \mathrm{d} y \mathrm{~d} z
$$

where $v$ is the $x$-component of the acoustic velocity and $S=l d$ is the rectangular cross section.

\subsection{Comparison between the two waves approaches}

In this section, the results between both methods are compared in the case of a street canyon (Fig. 1(b)) of fixed dimensions $l=d=10 \mathrm{~m}$, for the 
three chosen frequencies $f=62.5,125,250 \mathrm{~Hz}$.

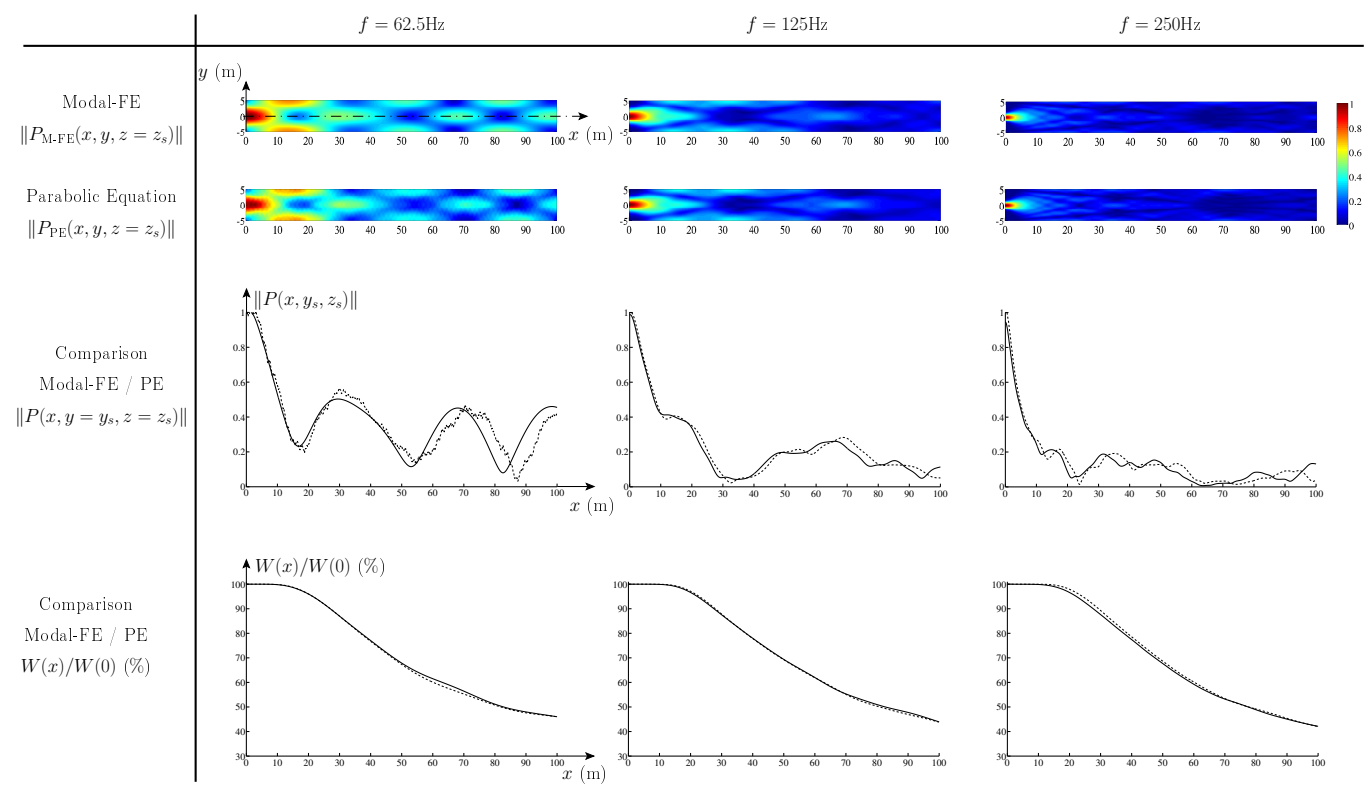

Figure 3: Comparison of the results between the two wave approaches in the case of a street canyon (Fig. 1(b)) of fixed width $l=10 \mathrm{~m}$ and height $d=10 \mathrm{~m}$ at three given frequencies $f=62.5,125,250 \mathrm{~Hz}$. For each frequency, the results are presented as, first, a horizontal map of the pressure field (modulus) at a height $z=z_{s}$ from the ground, second, the pressure field (modulus) along a line defined by $(y, z)=\left(y_{s}, z_{s}\right)(-$ : modal-FE / - : $\mathrm{PE}$ ), third, the decrease of the integrated indicator $W(x)$ function of $x$ (- : modal-FE / - - : PE).

First, the comparison concerns the pressure field. Fig. 3 shows horizontal maps of the pressure field (modulus) at a height $z=z_{s}$ from the ground. For each chosen frequency of the incident Gaussian beam, the sound maps computed from the two methods display very similar interference patterns. In particular, on the line defined by $(y, z)=\left(y_{s}, z_{s}\right)$, the solution are in good agreement at $f=125 \mathrm{~Hz}$. Nevertheless, at $f=62.5 \mathrm{~Hz}$ and $f=250 \mathrm{~Hz}$, one can see slight discrepancies due to phase shifts between both methods. On the one hand, for computation cost reasons at $f=250 \mathrm{~Hz}$, the mesh size in the modal-FE method is much more larger than the discretization step in the parabolic equation method. This relative sub-discretization of the transverse domain tends to stiff the problem which leads to a phase shift. 
On the other hand, at low frequency $(f=62.5 \mathrm{~Hz})$, the parabolic method propagates numerical artifacts due to the truncation of the Gaussian beam starter, which is larger than the street width. These numerical artifacts cause spurious oscillations and a phase shift.

Then, the comparison concerns the results obtained for the integrated indicator $W(x)$ defined in Eq. (10). Fig. 3 shows the decrease of the dimensionless value of $W(x) / W(x=0)$, for each studied case. Results obtained with both methods are almost the same. As a consequence, parametric studies in the following can be performed by one or the other method. The parabolic equation method is chosen because of its low computational cost.

\subsection{Effect of the street width}

The goal of this section is to compare the acoustic energy flux along the street computed for both "U-shaped" and "T-shaped" geometries of Fig. 1 for several frequencies and street widths. Results are summarized in Fig. 4. All curves exhibit a similar behavior: a flat phase in the near field followed by a monotonic decrease. The length of the flat phase directly depends on the Gaussian starter directivity and would not exist with an ideal omnidirectional starter. The acoustic energy flux decrease can by explained by the acoustic leakage across the street open ceiling. In all cases, the acoustic energy flux decrease is slower for the "T-shaped" geometry which shows that some acoustic energy is reflected at the opening of the street.

To simplify the reading of the results, the difference $\Delta_{W}$ between the acoustic flux in both geometries is given at $100 \mathrm{~m}$ from the source. One can see that, for a given street width, $\Delta_{W}$ decreases with the frequency. Otherwise, for a given frequency, $\Delta_{W}$ decreases with the street width. Interesting cases are those for whom the ratio $\eta=\frac{l}{\lambda}$ is constant (cases (a.3)-(b.2)-(c.1) or (a.2)-(b.1) or (b.3)-(c.2)) and where $\Delta_{W}$ is nearly the same. This observation tends to show that the dimensionless ratio $\eta$ is a significant parameter to quantify the contribution of the acoustic reflection at the opening.

In conclusion, if $\eta$ is too small, the approximation of total absorption at the opening is no longer verified: there exists a wave guiding effect between the ground and the open ceiling, in addition to the one between the façades. This result is not surprising if one consider the case $\eta \rightarrow 0$ corresponding to 


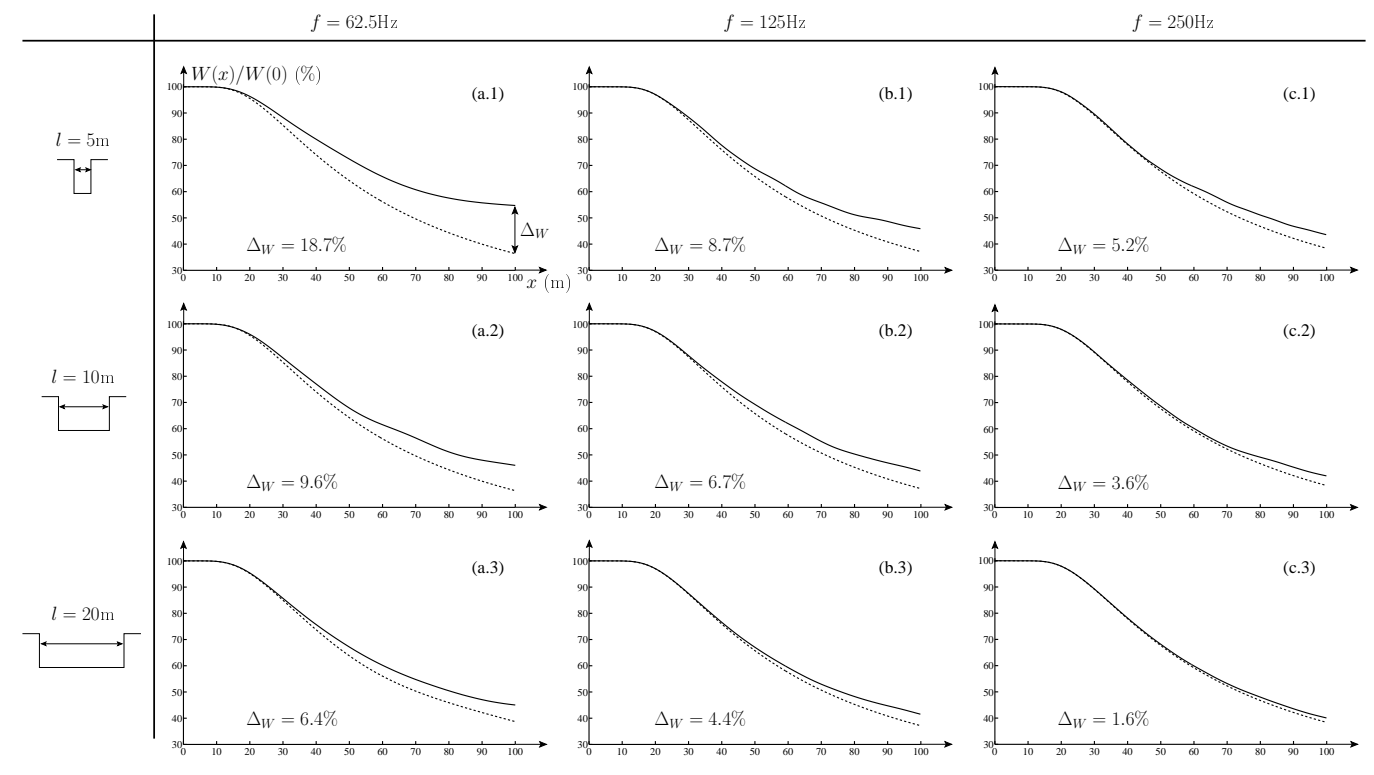

Figure 4: Acoustic energy flux decrease along the street. "U-shaped" (- - ) and "Tshaped" (-) geometries are compared for 3 frequencies $f=62.5,125,250 \mathrm{~Hz}$ and 3 street widths $l=5,10,20 \mathrm{~m}$.

total reflection as at the open extremity of a pipe.

\subsection{Effect of the source height}

In this section, we study the influence of the source height on the acoustic energy flux decrease along the street. Three locations of the source are studied: $z_{s}=0.1,1$ and $2 \mathrm{~m}$. These locations are representative of an equivalent source of a vehicle $\left(z_{s}=0.1 \mathrm{~m}\right)$, or of the human activity in urban environment $\left(z_{s}=1\right.$ and $\left.2 \mathrm{~m}\right)$. Results are summarized in Fig. 5. Three configurations are chosen. The first one (column 1) corresponds to a configuration where the guiding effect is significant $(l=5 \mathrm{~m}, f=62.5 \mathrm{~Hz})$. The second one (column 2) corresponds to an intermediate configuration $(l=10 \mathrm{~m}, f=125 \mathrm{~Hz})$. The third one (column 3$)$ corresponds to a configuration where the street open ceiling is almost totally absorbing $(l=20 \mathrm{~m}$, $f=250 \mathrm{~Hz})$.

First, the overall pattern of the decrease of $W(x) / W(0)$ can be discussed. 


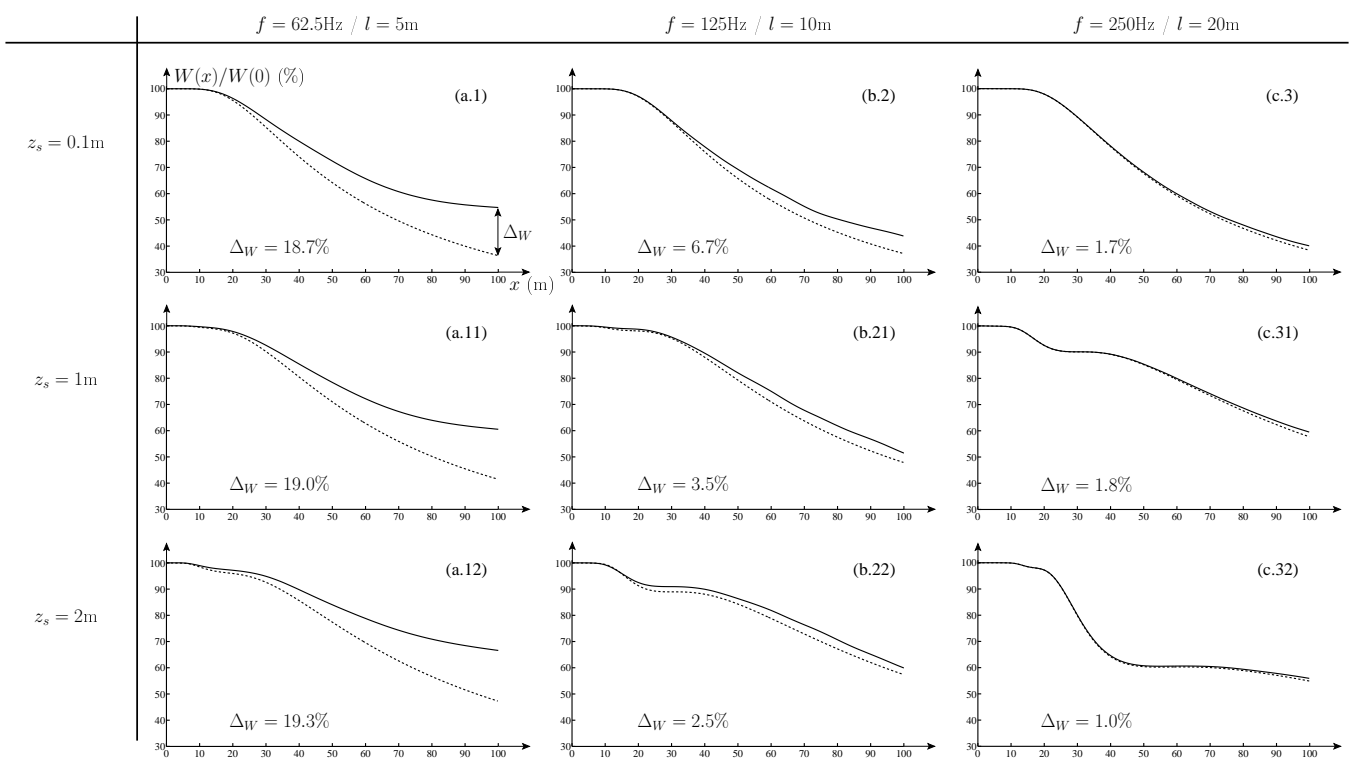

Figure 5: Acoustic energy flux decrease along the street."U-shaped" (- - ) and "T-shaped" $(-)$ geometries are compared for 3 frequencies $f=62.5,125,250 \mathrm{~Hz}$ and 3 source heights $z_{s}=0.1,1,2 \mathrm{~m}$.

When $z s<<\lambda$ (configurations (a.1), (a.11), (a.12), (b.2), (b.21), (c.3)), the same behavior as in section 3.3 is retrieved and $z_{s}$ has no significant effect. In configurations (b.22), (c.31) and (c.32), one can observe a local strong decrease, caused by reflection on the ground, followed by a slower decrease. Indeed, when $z_{s}$ is not small compared to $\lambda$, the ground effect results in a specular reflection that locally highly increases the $z$-component of the acoustic intensity at the top of the street and leads to strong radiation losses.. This phenomenon is notably very well pronounced in the range $20<x<40 \mathrm{~m}$ for the configuration (C.32).

Then, the results obtained for the $\Delta_{W}$ indicator can be discussed. In the intermediate case for which $f=125 \mathrm{~Hz}$ and $l=10 \mathrm{~m}$ (column 2 of Fig. 5), $\Delta_{W}$ decreases when $z_{s}$ increases: the open ceiling of the street tends to be totally absorbing when $z_{s}$ increases. This behavior can be explained by a modal representation of the acoustic field. Indeed, the more the source is elevated, the more the radiating modes are excited (mode shown in Fig. 2(c), for example). Furthermore, at $f=125 \mathrm{~Hz}$, these radiating modes, in addition to 


\begin{tabular}{|l||c|c|c|}
\hline Material & 62.5 & 125 & 250 \\
\hline Asphalt & 0.00 & 0.00 & 0.01 \\
Brickwork, unglazed & 0.03 & 0.03 & 0.03 \\
Grass Ground & 0.04 & 0.06 & 0.10 \\
$16 \mathrm{~mm}$ wood at $4 \mathrm{~cm}$ framework & 0.20 & 0.18 & 0.12 \\
\hline
\end{tabular}

Table 1: Absorption coefficients (from [19]) used in the calculations. The impedances of asphalt and grass have been calculated using the Delany and Bazley impedance model [20].

the confined modes, significantly participate to the field, which enhances the radiation losses. In the case where $f=62.5 \mathrm{~Hz}$ and $l=5 \mathrm{~m}$, the source height has no significant effect. At a such low frequency, whatever the modal development of the source, only one mode, confined within the street (mode shown in Fig. 2(a)), significantly participates to the propagation of the field. For the configuration $f=250 \mathrm{~Hz}$ and $l=5 \mathrm{~m}$, we also observe that the source height has no significant effect on $\Delta_{W}$. This case corresponds to a case for which the open ceiling is almost perfectly absorbing, whatever the source height.

\subsection{Effect of the wall absorption}

In this section, absorption at the walls and the ground are introduced. the effects of wall and ground absorptions on the leakage are examined. This study aims to i) examine the effects of wall and ground absorptions on the leakage through the open ceiling of the street, ii) evaluate the relative effects of this additional loss mechanism compared to the leakage. At each frequency $f=62.5,125$ and $250 \mathrm{~Hz}, 3$ absorbing configurations are tested for both "Ushaped" and "T-shaped" geometries: a hard walls configuration, a reasonable absorbing configuration with asphalt ground and unglazed brickwork walls, and a relative highly absorbing configuration (regarding the frequency range of interest and the typical materials used in urban spaces) with grassy ground and wood framework walls. Tab. 1 summarizes the absorption coefficients used in calculations [19]. The $62.5 \mathrm{~Hz}$ absorption coefficients of the brickwork and the wood framework are arbitrarily fixed because they are not available in the literature. In this study, the street width is $l=10 \mathrm{~m}$ and the source height is $z_{s}=0.1 \mathrm{~m}$.

Results are summarized in Fig. 6. The decreases of $W(x)$ display an ex- 


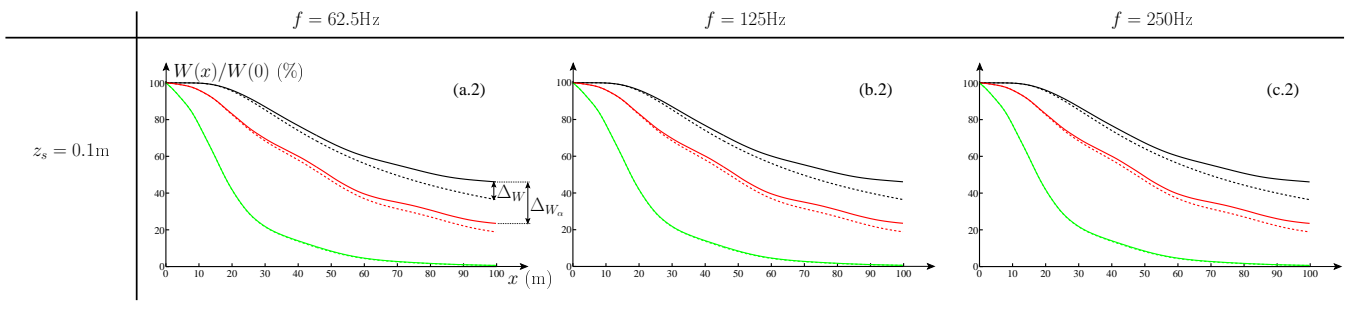

Figure 6: Acoustic energy flux decrease along the street. "U-shaped" (---) and "T-shaped" (-) geometries are compared for 3 frequencies $f=62.5,125,250 \mathrm{~Hz}$ and 3 absorbing configurations: rigid walls configuration (black), reasonable absorbing configuration with asphalt ground and unglazed brickwork walls (red), highly absorbing configuration with grassy ground and wood framework as walls (green).

pected behavior: the more absorbent are the walls and ground, the faster is the decrease. The comparison between the "U-shaped" and "T-shaped" geometries shows that, at a given frequency, $\Delta_{W}$ decreases when the wall absorption increases (Tab. 2). $\Delta_{W}$ is even almost zero for the highly absorbing configuration. In this condition, there is no need to use the "T-shaped" geometry. Indeed, the absorption at the walls tend to soften the acoustic impedance break at the top of the street and the guiding effects between the ground and the open ceiling vanish.

\begin{tabular}{|l|ccc|}
\hline$f(\mathrm{~Hz})$ & 62.5 & 125 & 250 \\
\hline$\Delta_{W}$ (hard wall) (\%) & 10 & 7 & 4 \\
\hline$\Delta_{W}$ (reasonably absorbing)(\%) & 5 & 4 & 3 \\
\hline$\Delta_{W}$ (highly absorbing) (\%) & 0 & 0 & 1 \\
\hline$\Delta_{W_{\alpha}}(\%)$ & 23 & 15 & 11 \\
\hline
\end{tabular}

Table 2: Values of the indicators $\Delta_{W}$ and $\Delta_{W_{\alpha}}$ at the three studied frequencies.

To evaluate the relative effects between the absorption at the walls and the leakage through the open ceiling, a new indicator $\Delta_{W_{\alpha}}$, analogous to $\Delta_{W}$, is required. $\Delta_{W_{\alpha}}$ is defined as the difference at $x=100 \mathrm{~m}$ from the source between the acoustic flux for the hard walls and the reasonably absorbing configurations in the "T-shaped" geometry $\left(\Delta_{W_{\alpha}}\right.$ is graphically represented in the left plot of Fig. 6). Results in Tab. 2 show that the assumption of hard walls leads to an overestimation of the energy flux of about $\Delta_{W_{\alpha}}=23 \%$ at 
62.5 Hz while the hypothesis of perfectly absorbing ceiling leads to an underestimation of $\Delta_{W}=10 \%$ (in the hard walls configuration). The situation is similar for the other studied frequencies. Thus, we see that the error made by assuming a perfectly absorbing ceiling is not negligible compared to the error made by considering perfectly reflecting walls.

\section{Conclusions}

In this paper, we propose to use two wave methods to study the acoustic propagation along a street. The work focuses on the radiation condition at the opening of the street. First, the comparison of these methods shows a very good agreement.

Next the parametric studies point out that the assumption of total absorption at the street open ceiling is not valid in all situations. Indeed, the dimensionless ratio $\eta=\frac{l}{\lambda}$ seems to drive the acoustic reflection at the street open ceiling. For example, one can arbitrarily determine the following criterion: the total absorption hypothesis is no longer valid if it leads to a leakage difference $\Delta_{W}$ higher than $5 \%$ at $100 \mathrm{~m}$ from the source. Results of Fig. 4 show that the hypothesis is not valid below $125 \mathrm{~Hz}$ for a $20 \mathrm{~m}$ wide street or below $250 \mathrm{~Hz}$ for a $10 \mathrm{~m}$ wide street.

It is also shown that the source height $z_{s}$ has no significant effects on $\Delta_{W}$ except when $z_{s}$ is not small compared to the wavelength. In this condition, $\Delta_{W}$ tends to be reduced when $z_{s}$ increases.

At least, considering absorbing walls and ground tends to reduce $\Delta_{W}$. However, the results show that, in the case of reasonable absorbing configuration, the assumption of perfectly absorbing ceiling leads to an error of the same order of magnitude than the assumption of perfectly hard walls.

Many improvements can be provided to this work. Concerning the methods used, works are in progress to bring down the numerical cost of the modalFE method by performing more advanced FEM calculations of the modes. This will allow to perform calculations for higher frequencies $(f \geq 250 \mathrm{~Hz})$. Furthermore, the study of acoustic leakage through the open ceiling is led 
by the acoustic energy flux calculus. This physical quantity may not be appropriated with perceptive sensations. Nevertheless, this integrated quantity is convenient to avoid interferences (see sound maps in Fig. 3) which make the decrease estimation difficult. Another indicator could be the averaged acoustic level at ears height in an octave band. An appropriate number of harmonic calculus will be needed to estimate the averaged field in the octave band of interest. Whatever the criterion used, it also will be appreciable to work with more realistic street geometries including building facades irregularities. This issue will require either to improve the PE model to take into account sound scattering from the walls in all directions, or to use more adapted numerical approach. At least, it would be interesting to study the impact of the micro meteorological effects as refraction or turbulence on the reflection at the open ceiling.

\section{5. acknowledgments}

This work has received support from the National French Agency for Research reference (reference of the project: ANR-JCJC-3019-01) and from the Regional council of the Pays de La Loire.

\section{References}

[1] O. Richoux, C. Ayrault, A. Pelat, S. Félix, and B. Lihoreau. Effect of the open roof on low frequency acoustic propagation in street canyons. Appl. Acoust., 71:731-738, 2010.

[2] H.G. Jonasson. Acoustical source modelling of road vehicles. Acta Acustica united with Acustica, 93(2):173-184, 2007.

[3] A. Can, L. Leclercq, J. Lelong, and D. Botteldooren. Traffic noise spectrum analysis: Dynamic modeling vs. experimental observations. Appl. Acoust., 71:764-770, 2010.

[4] ISO 9613-2 Acoustics - Attenuation of sound during propagation outdoors - Part 2: General method of calculation, 1996.

[5] J.S. Bradley. A study of traffic noise around buildings. Acustica, 38:247252, 1977. 
[6] K. K. Lu and K. M. Li. The propagation of sound in narrow street canyons. J. Acoust. Soc. Am., 112(2):537-550, 2002.

[7] J. Kang. Sound propagation in street canyons : Comparison between diffusely and geometrically reflecting boundaries. J. Acoust. Soc. Am., 107(3):1394-1404, March 2000.

[8] T. Le Pollès, J. Picaut, S. Colle, M. Bérengier, and C. Bardos. Soundfield modeling in architectural acoustics by a transport theory: Application to street canyons. Phys. Rev. E, 72(4):046609, 2005.

[9] J.-P. Bérenger. A perfectly matched layer for the absorption of electromagnetics waves. J. Comp. Phys., 114(2):185-200, 1994.

[10] M. Hornikx, R. Waxler, and J. Forrsén. The extended fourier pseudospectral time-domain method for atmospheric sound propagation. $J$. Acoust. Soc. Am., 128(4):1632-1646, 2010.

[11] T. Van Renterghem and D. Botteldooren. The importance of roof shape for road traffic noise shielding in the urban environment. J. Sound Vib., 329(9):1422--1434, 2010.

[12] B. Lihoreau and O. Richoux. A 3D parabolic equation code for street canyon propagation. In Proc. of Euronoise, 2009.

[13] R. Bullen and F. Fricke. Sound propagation in a street. J. Sound Vib., 46(1):33-42, 1976.

[14] A. Pelat, S. Félix, and V. Pagneux. On the use of leaky modes in open waveguides for the sound propagation modeling in street canyons. $J$. Acoust. Soc. Am., 126(6):2864-2872, 2009.

[15] F.D. Tappert. The parabolic approximation. In J. B. Keller and J. S. Papadakis, editors, Wave propagation in underwater acoustics. SpringerVerlag, New-York, 1977.

[16] A. Bamberger, B. Engquist, L. Halpern, and P. Joly. Higher order paraxial wave equation approximation in heterogeneous media. SIAM J. Appl. Math., 48(1):129-154, 1988. 
[17] M.D. Collins and S.A. Ching-Bing. A three dimensional parabolic equation mode that includes the effect of rough boundaries. J. Acoust. Soc. Am., 87(3):1104-1109, 1990.

[18] A. Pelat, S. Félix, and V. Pagneux. A coupled modal-finite element method for the wave propagation modeling in irregular open waveguides. J. Acoust. Soc. Am., 129(3):1240-1249, 2011.

[19] M. Hornikx and J. Forssén. Noise abatement schemes for shielded canyons. Appl. Acoust., 70:267-283, 2009.

[20] M.E. Delany and E.N. Bazley. Acoustical properties of fibrous absorbant materials. Appl. Acoust., 3(2):105-116, 1970. 